\title{
Seminal fluid protein genes of the brown planthopper, Nilaparvata lugens
}

\author{
Bing Yu, Dan-Ting Li, Jia-Bao Lu, Wen-Xin Zhang and Chuan-Xi Zhang ${ }^{*}$ (i)
}

\begin{abstract}
Background: Seminal fluid proteins (SFPs) are produced mainly in the accessory gland of male insects and transferred to females during mating, in which they induce numerous physiological and post-mating behavioral changes. The brown plant hopper (BPH), Nilaparvata lugens, is an economically important hemipterous pest of rice. The behavior and physiology of the female of this species is significantly altered by mating. SFPs in hemipteran species are still unclear.

Results: We applied high-throughput mass spectrometry proteomic analyses to characterize the SFP composition in N. lugens. We identified 94 putative secreted SFPs, and the expression levels of these proteins was determined from the male accessory gland digital gene expression database. The 94 predicted SFPs showed high expression in the male accessory gland. Comparing N. lugens and other insect SFPs, the apparent expansion of N. lugens seminal fluid trypsins and carboxylesterases was observed. The number of $N$. lugens seminal fluid trypsins (20) was at least twice that in other insects. We detected 6 seminal fluid carboxylesterases in N. lugens seminal fluid, while seminal fluid carboxylesterases were rarely detected in other insects. Otherwise, new insect SFPs, including mesencephalic astrocyte-derived neurotrophic factor, selenoprotein, EGF (epidermal growth factor) domain-containing proteins and a neuropeptide ion transport-like peptide were identified.
\end{abstract}

Conclusion: This work represents the first characterization of putative SFPs in a hemipeteran species. Our results provide a foundation for future studies to investigate the functions of SFPs in N. lugens and are an important addition to the available data for comparative studies of SFPs in insects.

Keywords: Nilaparvata lugens, Seminal fluid protein, Proteome, UPLC/MS/MS

Abbreviations: $\mathrm{BPH}$, Brown planthopper; $\mathrm{CB}$, Copulatory bursa; CDS, Coding sequence; DGE, Digital gene expression; EGF, Epidermal growth factor; FRT, Female reproductive tract; ITPL, Ion transport peptide like; MADF, mesencephalic astrocyte-derived neurotrophic factor; MAG, Male accessory gland; MRT, Male reproductive tract; RNAi, RNA interference; RPKM, Reads Per Kilo bases per Million mapped Reads; RT-qPCR, Reverse-transcription quantitative PCR; SFPs, Seminal fluid proteins; SR, Seminal receptacle; TE, Testes; VD, vas deferen

\section{Background}

Insect seminal fluid proteins (SFPs) are important for fertilization and are weapons for males in sexual competition, such as manipulating post-mating physiological and behavioral changes in females [1]. SFPs have complex structures and perform a diversity of functions [2]. Under natural selection and selection by females, and under male competition [2], the rapid evolution of SFPs

\footnotetext{
* Correspondence: chxzhang@zju.edu.cn

State Key Laboratory of Rice Biology and Ministry of Agriculture Key
Laboratory of Agricultural Entomology, Institute of Insect Science, Zhejiang

State Key Laboratory of Rice Biology and Ministry of Agriculture Key
Laboratory of Agricultural Entomology, Institute of Insect Science, Zhejiang University, Hangzhou 310058, China
}

(c) 2016 The Author(s). Open Access This article is distributed under the terms of the Creative Commons Attribution 4.0 International License (http://creativecommons.org/licenses/by/4.0/), which permits unrestricted use, distribution, and reproduction in any medium, provided you give appropriate credit to the original author(s) and the source, provide a link to the Creative Commons license, and indicate if changes were made. The Creative Commons Public Domain Dedication waiver (http://creativecommons.org/publicdomain/zero/1.0/) applies to the data made available in this article, unless otherwise stated. species exhibit significant diversity, presumably enabling a variety of reproductive strategies. Week-long refractoriness toward further copulation and enhanced egg laying levels are generated by the seminal fluid sex peptide (Acp70A) pathway in Drosophila melanogaster, and sperm is required in this process [4-6]. An unknown mechanism leads to life-long behavioral changes in mosquitoes such as Anopheles gambiae, and sperm is not required [7]. The female behavior and physiology of multiple mating social insects are apparently unaffected by a single copulation, but SFPs may respond to the 
long-term storage of sperm and sperm competition after copulation $[8,9]$.

The brown planthopper (BPH), Nilaparvata lugens Stål (Hemiptera: Delphacidae), is one of the most serious insect pests of rice in Asia [10]. Asian countries have continually experienced serious outbreaks of $\mathrm{BPH}$ although new BPH-resistant rices, new insecticides, as well as integrated pest management programs are used. Mated BPH females display stimulated egg laying levels [11] and almost life-long refractoriness to further insemination [12]. The sex peptide model, as used to describe post-mating behavior in D. melanogaster, may not provide a reasonable explanation for post-mating behavior in $N$. lugens. At present, chemical control remains the first choice for $N$. lugens management [13]. Seminal fluid might play a part in the rapid establishment of drug resistance. Insecticide (triazophos and deltamethrin)treated male $N$. lugens had higher protein content than untreated males; treated males also transferred more SFPs to mated females $[14,15]$.

Proteomic approaches to elucidating the function of SFPs have been carried out on several insect species, including Apis mellifera [8], D. melanogaster, D. simulans, D. yakuba [16], Tribolium castaneum [17], Aedes aegypti [18], Aedes albopictus [19], Teleogryllus oceanicus [20], Heliconius erato, and $H$. melpomene [21]. Proteomic research on SFPs has not been performed for any hemipterous species to date, such as $N$. lugens. Furthermore, the functions of insect SFPs have been poorly studied in insects other than $D$. melanogaster, despite major differences in reproductive physiology exist between species. More seminal protein information from multiple insects could provide more insights into the evolutionary patterns of reproductive traits [22]. As more is learned about the reproductive biology of specific arthropods, their SFPs may provide tools or targets for the control of disease vectors and agricultural pests [22]. The $N$. lugens seminal fluid proteome could benefit research into the reproductive physiology of $N$. lugens that uses tools such as RNA interference (RNAi). Illustrating the molecular interactions between SFPs and $N$. lugens females may aid researchers in identifying molecular targets for pest control, as the regulation of female behaviors after mating appears to be long-lasting in $N$. lugens.

Recently, the whole genome sequences and gene annotation information for $N$. lugens were described [10]. Gene expression information regarding developmental stages, wing dimorphism, sex differences, and tissues was collected using next-generation high-throughput Illumina technology [13, 23-26]. The male reproductive tract (MRT) of $N$. lugens comprises two testes (TE), two vas deferens (VD), two male accessory glands (MAGs), and one ejaculatory duct (Fig. 1). Sperm are produced by the TE, and SFPs are produced primarily by the MAGs. In this study, transcriptomic analysis of the $N$. lugens MRT was performed, and gene expression information concerning the MAG was obtained using a tag-based digital gene expression (DGE) system. We used UPLC/ MS/MS to identify the transferred SFPs of $N$. lugens.

\section{Methods}

Insects

The $N$. lugens strain was originally collected from a rice field located in the Huajiachi Campus of Zhejiang University in Hangzhou, China. The insects used in this experiment were the offspring of a single female. Insects were reared on rice seedlings at $28{ }^{\circ} \mathrm{C}$ (Xiu shui 128) under a 12:12 h light: dark photoperiod.

\section{Preparation of $N$. lugens MRT transcriptome database}

$N$. lugens males were anesthetized on ice for $20 \mathrm{~min}$ and dissected under a Leica S8AP0 stereomicroscope. The whole MRT (including the TE, VD, and MAGs) (Fig. 1) were isolated and quickly washed in a diethylpyrocarbonate

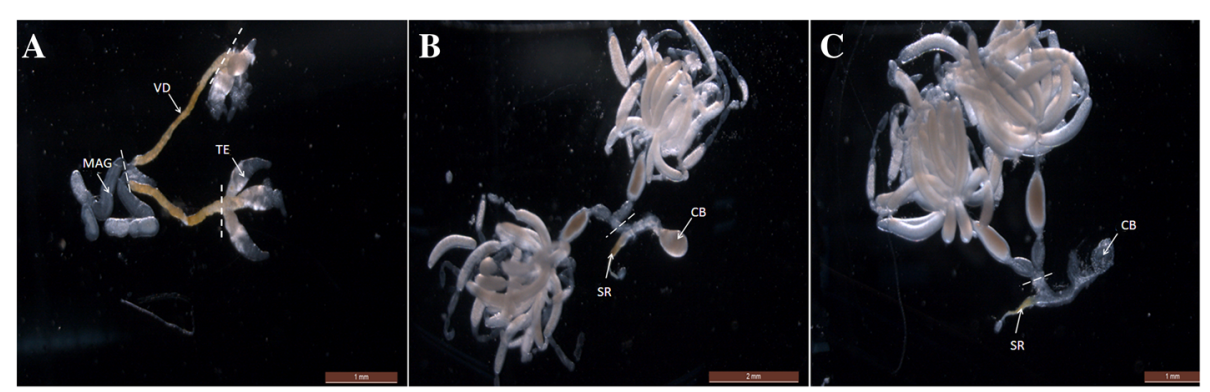

Fig. 1 Tissues dissected for sample preparation. a Male reproductive tract (MRT). The whole MRT and dissections of MRT (including TE, VD, and MAGs) dissected from 40 males were collected for RT-qPCR. MAGs dissected from 50 males were collected for MAG protein sample preparation. Each tissue was dissected from the dotted line. $\mathbf{b}$ Mated female reproductive tracts. Copulatory bursas (CB) and seminal receptacles (SR) dissected from 50 mated females were collected for mated-FRT protein sample preparation. Each tissue was dissected from the dotted line. c Unmated FRTs. The whole FRT dissected from 40 unmated females were collected for RT-qPCR. Copulatory bursas (CB) and seminal receptacles (SR) dissected from 50 females were collected for unmated-FRT protein sample preparation. Each tissue was dissected from the dotted line 
(DEPC)-treated phosphate-buffered saline (PBS) solution (137 mM NaCl, $2.68 \mathrm{mM} \mathrm{KCl,} 8.1 \mathrm{mM} \mathrm{Na} \mathrm{HPO}_{4}$, and $1.47 \mathrm{mM} \mathrm{KH}_{2} \mathrm{PO}_{4}$ at $\mathrm{pH} 7.4$ ) and were immediately frozen at $-80{ }^{\circ} \mathrm{C}$. The MRT sample was used for transcriptome and DGE sequencing, and the MAG sample was used for DGE sequencing.

Total RNA was isolated from $N$. lugens MRT and MAG using TRIzol reagent (Invitrogen, Carlsbad, CA, USA) following the manufacturer's instructions. Sequencing and assembly of transcriptome reads, including DGE library reads, was performed using Illumina HiSeq $^{\text {тм }} 2000$ and Trinity (v2012-10-05), respectively, and the annotation of unigenes were performed as described previously [23]. The longest assembled transcripts of each gene were taken as unigenes. The readcount of each unigenes was normalized to RPKM (Reads Per Kilo bases per Million mapped Reads) to display the expression level of each unigene. The coding sequence (CDS) of each unigene was analyzed using blastx and estscan (3.03). The generated peptide database was used to support the proteomic analysis.

\section{Seminal fluid protein sample preparation}

Seminal fluid samples were collected from males and mated females, and soluble protein samples were collected from unmated females (all individuals were 4-7 days post-eclosion). Mated females were obtained by placing one female in a glass tube containing a rice seedling with one male for $2 \mathrm{~h}$. The female copulatory bursa (CB) and seminal receptacle (SR) (Fig. 1) were dissected in PBS solution and squeezed using grinding rod in $100 \mu \mathrm{l}$ PBS with $1 \%$ protease inhibitor cocktail (Thermo, USA). Reproductive tracts from $\approx 50$ females were pooled for each biological replicate. MAGs (Fig. 1) were dissected using the same method, and MAGs from $\approx 50$ males were collected for each biological replicate. Samples were centrifuged at $12,000 \mathrm{rpm}$ for $20 \mathrm{~min}$ at $4{ }^{\circ} \mathrm{C}$. The supernatant was transferred to a separate tube and stored at $-80{ }^{\circ} \mathrm{C}$. Three replicates were prepared for each kind of sample including the MAG, the mated-female reproductive tract (FRT) and the unmated-FRT.

A filter aided sample preparation (FASP) method was used for the preparation of samples [27]. Samples were added to $3 \mathrm{kD}$ ultrafiltration centrifuge tubes (Millipore), and centrifuged at $14000 \mathrm{~g}$ for $20 \mathrm{~min}$. $100 \mu \mathrm{l}$ of UA solution [8 M Urea (Sigma), 0.1 M Tris/HCl pH 8.5, $1 \%$ EDTA (Thermo), $1 \%$ protease inhibitor Cocktail (Thermo)], and centrifuged at $14000 \mathrm{~g}$ for $20 \mathrm{~min}$; this step was repeated twice. $2 \mu$ DTT (Sigma) (200 mM) was added. Samples were vortexed for $1 \mathrm{~min}$ and incubated at $37{ }^{\circ} \mathrm{C}$ for $1 \mathrm{~h} .20 \mu \mathrm{l}$ iodoacetamide (Sigma) (200 $\mathrm{mM})$ was then added. Samples were vortexed for $1 \mathrm{~min}$ and incubated at $25{ }^{\circ} \mathrm{C}$ for $1 \mathrm{~h}$ in the dark.
Samples were centrifuged at $14000 \mathrm{~g}$ for $20 \mathrm{~min}$. To each sample, $100 \mu \mathrm{l}$ UA was added. Samples were then centrifuge at $14000 \mathrm{~g}$ for $20 \mathrm{~min}$; this step was repeated once. $200 \mu \mathrm{l} \mathrm{NH}_{4} \mathrm{HCO}_{3}$ (Sigma) (0.05 M) was added, and samples were centrifuged at $14000 \mathrm{~g}$ for $20 \mathrm{~min}$; this step was repeated twice. The remaining sample was moved into a $10 \mathrm{kD}$ ultrafiltration centrifuge tube, and $40 \mu \mathrm{NH}_{4} \mathrm{HCO}_{3}(0.05 \mathrm{M})$ and trypsin (Promega) $(5 \mu \mathrm{g}$ in total) were added. Samples were incubated at $30{ }^{\circ} \mathrm{C}$ for $12 \mathrm{~h}$, and then centrifuged at $14000 \mathrm{~g}$ for $20 \mathrm{~min}$. $40 \mu \mathrm{NH}_{4} \mathrm{HCO}_{3}(0.05 \mathrm{M})$ was added; then samples were centrifuged at $14000 \mathrm{~g}$ for $30 \mathrm{~min}$. Filtered liquid was removed into a $1.5 \mathrm{ml}$ centrifuge tube and dehydrated in a vacuum freeze-drying device. Dehydrated samples were dissolved in $25 \mu \mathrm{l} 0.1 \%$ formic acid (Sigma). The concentrations of the dissolved peptide solutions were analyzed by A280 absorption using a NanoDrop UV-vis spectrophotometer (Thermo Fisher Scientific, Waltham, Massachusetts, USA).

\section{UPLC/MS/MS methods and data analyses}

The peptide mixtures were injected onto the trap column at a flow rate of $10 \mu \mathrm{l} / \mathrm{min}$ for $2 \mathrm{~min}(2 \mu \mathrm{g})$ using a Thermo Scientific Easy nanoLC 1000. The trap was equilibrated at a maximum pressure of 500 bar for $12 \mu \mathrm{l}$, followed by column equilibration at a maximum of 500 bar for $3 \mu \mathrm{l}$ before beginning the gradient elution of the column. The samples were subsequently eluted using the following five-step linear gradient (A: ddH2O with $0.1 \%$ formic acid,B: ACN with $0.1 \%$ formic acid): 0 $10 \mathrm{~min}, 3-8 \% \mathrm{~B} ; 10-120 \mathrm{~min}, 8-20 \% \mathrm{~B} ; 120-137 \mathrm{~min}$, 20-30\% B; 137-143 min, 30-90 \% B; and 143-150 min, $90 \% \mathrm{~B}$. The column flow was maintained at $250 \mathrm{~nL} / \mathrm{min}$. The chromatographic system was composed of a trapping column (75 $\mathrm{mm} \times 2 \mathrm{~cm}$, nanoviper, $\mathrm{C} 18,3 \mu \mathrm{M}, 100 \AA)$ and an analytical column $(50 \mu \mathrm{m} \times 15 \mathrm{~cm}$, nanoviper, C18, $2 \mu \mathrm{M}, 100 \AA$ ). Data collection was performed using a Thermo LTQ-Orbitrap Velos Pro equipped with a Nanospray Flex ionization source and a FTMS (Fourier transform ion cyclotron resonance mass spectrometry) analyzer combined with a Thermo LTQ-Orbitrap Elite equipped with an ion trap analyzer. The parameters for FTMS were as follows: Data collection at $60 \mathrm{~K}$ for the full MS scan, positive polarity, data type profile, and then proceeded to isolate the top 20 ions for MS/MS by CID $(1.0 \mathrm{~m} / \mathrm{z}$ isolation width, $35 \%$ collision energy, 0.25 activation $\mathrm{Q}, 10 \mathrm{~ms}$ activation time). The scan range was set as $300 \mathrm{~m} / \mathrm{z}$ first mass and $2000 \mathrm{~m} / \mathrm{z}$ last mass. The parameters for the ion trap analyzer were the normal mass range, rapid scan rate, and centroid data type.

A SEQUEST HT search engine configured with a Proteome Discoverer 1.4 workflow (Thermo Fischer Scientific, Bremen, Germany) was used for mass spectrometer data analyses. An $N$. lugens MRT peptide 
database generated from transcriptome unigene sequences database containing 17902 sequences were configured with SEQUEST HT for dataset searches. The search parameters included $10 \mathrm{ppm}$ and $0.8 \mathrm{Da}$ mass tolerances for MS and MS/MS, respectively, trypsin as the proteolytic enzyme with two allowed missed cleavages, oxidation and deamidated as dynamic modifications, and carbamidomethyl as a static modification. Furthermore, the peptides were extracted using high peptide confidence. $1 \%$ FDR (False discovery rate) was calculated using a decoy database by searching both the MRT peptide sequence and the decoy database.

\section{Identification of seminal fluid proteins of $N$. lugens}

High confidence proteins were identified with the following standards: 1) Proteins identified from more than two samples (proteins derived from at least two MAG samples, two unmated-FRT samples and two mated-FRT samples) were predicted to be "true" detected proteins. 2) Seminal fluid proteins must have been identified from both MAG and mated-FRT samples. 3) We tested for predicted secretion signal sequences of detected proteins using SignalP 4.1 (www.cbs.dtu.dk/services/SignalP/). Some sequences had a "bad" coding sequence CDS prediction (Lost in N-terminal), in which the signal peptide was not be predicted from the sequence. We repredicted CDS sequences for proteins with no signal peptide using ESTScan (http://myhits.isb-sib.ch/cgi-bin/ estscan) from unigene nucleotide sequences, and performed Signalp detection with new predicted CDS sequences for improved signal peptide detection. Proteins possessing a signal peptide were considere $d$ to be secreted proteins. 4) Some proteins did not possess a signal peptide. Proteins without signal peptides that were not detected in unmated-FRT samples and showed male-specific expression (an analysis of the male-specific expression of unigenes was performed as described previously [13]) were also predicted to be secreted. 5) In addition, other proteins that were not predicted to be secreted and had homologues in the SFPs of other insects were classied as unconfirmed SFPs.

\section{Annotation of seminal fluid proteins and comparison with other insects}

In addition to machine annotation, we performed a manual annotation for the sequences detected. Blast results from NCBI, conserved domains, and GO terms were used in combination to annotate proteins. Brief descriptions from NCBI, SMART (http://smart.emblheidelberg.de/) descriptions of conserved domains, and functional descriptions of gene names from UniProtKB (http://www.uniprot.org/help/uniprotkb) were used to classify the functions of each sequence. Based on these matches, proteins were classified into one of the following categories: cell structure (including cell structure proteins and their binding proteins), metabolism, protein modification machinery, proteolysis regulators (proteases and protease inhibitors), signal transduction (including hormones), transporters and protein export machinery, and RNA and protein synthesis (transcription factors, transcription machinery, and protein synthesis enzymes). Proteins that were classified into different categories were classified as "other" (including salivary proteins, chitin binding proteins, binding proteins, proteasome machinery, protein kinases, ubiquitination pathway proteins, protein phosphatases, and oxidoreductases). Proteins that were not assigned a function were classified as "unknown".

Seminal fluid proteome sequences of D. melanogaster, A. aegypti, A. albopictus, A. mellifera and Homo sapien [28] were chosen for comparison with SFPs of $N$. lugens. SFP sequences of $D$. melanogaster were extracted from Flybase (http://flybase.org/) using IDs given from reference [16]. SFPs sequences of $A$. aegypti were extracted from Ensembl Metazoa (http://metazoa.ensembl.org/ info/website/ftp/index.html) using IDs given from reference [18]. SFP sequences of $A$. albopictus were directly given by reference [19]. SFPs sequences of $A$. mellifera were extracted from NCBI (http://www.ncbi.nlm.nih. gov/sites/batchentrez) using IDs given from reference [8]. Signal peptides of these proteins were identified as mentioned in 2.5. And prediction of conserved domains of predicted protein domains was using the Batch Web CD-Search Tool (http://www.ncbi.nlm.nih.gov/Structure/ bwrpsb/bwrpsb.cgi). N. lugens SFPs possessing the same conserved domain with other insect SFPs were marked as "Domain". The rest proteins with blastp (Evalue $<10^{-5}$ ) hits with other insect SFPs were marked as "Blast". The same method was used for comparison of SFPs between insect species.

To locate the detected proteins in the $N$. lugens genome scaffold sequences, we run a megablast with Evalue $<10^{-20}$, and indentity $>95 \%$ between detected proteins and scaffold sequences.

\section{Phylogenetic analysis}

The functional serine protease domains of the $N$. lugens seminal fluid trypsins were aligned with seminal fluid trypsins of other insect species using the ClustalX program. The phylogenetic tree was constructed by the maximum likelihood (ML) method using the program Mega 5.05 (http://www.megasoftware.net/). Homologous relationships were determined using bootstrap analysis with 1000 replications.

Reverse-transcription quantitative PCR (RT-qPCR) analysis MRT, FRT, and dissections of MRT (including testes, vas deferens, and male accessory glands) (Fig. 1) were 
dissected from males (4-7 days post-eclosion). As the mRNA quantity of an individual tissue is extremely low, tissues dissected from 40 individuals were pooled into each tissue sample, respectively. RT-qPCR was performed according to the method of [29]. Primers used in RT-qPCR for the tissue specific expressions of seminal fluid protein genes are given in Additional file 1: Table S1.

\section{Results}

\section{MRT transcriptome sequencing and assembly}

Illumina sequencing produced 2.59 GB nucleotides. The quality of this transcriptome sequence was high, with a Q20 percentage (the percentage of sequences with a sequencing error rate of $0.03 \%$ ) and GC content of $98.21 \%$ and $40.08 \%$, respectively. These short reads were assembled into 57568 transcripts with a mean length of 741 bp. Ultimately, we obtained 37443 unigenes with a mean size of $641 \mathrm{bp}$ and lengths ranging from 201 to $9670 \mathrm{bp}$. Annotation of these sequences revealed that 13089 (35\%) sequences were annotated in the NR database, 10156 (27\%) sequences were annotated in the SwissProt database, 10589 (28 \%) sequences were annotated in the GO database, and 8301 (22\%) sequences were annotated in the KOG database. Of these, 523 sequences showed male-specific expression. A peptide database with 17902 sequences was generated as a query database for the raw data of the proteome (Table 1).

Two DGE libraries from the MRT and MAG were also sequenced, generating approximately $0.64 \mathrm{~GB}$ clean tags for each library. Among the clean tags, approximately $92 \%$ of sequences could be mapped to unigenes in each library. A total of 33798 unigenes expressed in the MRT had a RPKM value $>0.3 ; 19772$ unigenes expressed in the MAG had a RPKM value > 0.3 (Table 1). The MRT transcriptome yielded a peptide database used in proteome sequencing, and the

Table 1 Summary of transcriptome sequence datasets of the $\mathrm{BPH}$ reproductive tract

\begin{tabular}{ll}
\hline Group & Number of unigenes \\
\hline Total unigene & 37443 \\
MRT expression & 33798 \\
MAG expression & 19772 \\
Male specific expressed & 523 \\
Obtained peptide database & 17902 \\
\hline
\end{tabular}

The longest assembled transcripts of each gene were taken as unigenes. Only unigenes with RPKM value larger than 0.3 were counted as expressed. Owing to the accomplishment of the transcriptomic sequencing of the differences between the $N$. lugens development and sex genes in our previous study [11], we are able to use the predicted SFP genes as reference sequences to map the transcriptomic datasets and to analyze the expression sex-specific genes. The coding sequence (CDS) of each unigene was analyzed using blastx and estscan (3.03). The generated peptide database was used in proteome query
MRT and MAG DGE databases provided the expression levels of detected protein-coding genes in the MRT and MAG.

\section{Proteins transferred to females during mating}

We identified a total of 218 putative SFPs from both the MAG and mated-female reproductive tract (FRT) samples. Of these, 65 sequences were not detected in unmated-FRT samples and showed male-specific expression. Fifty-five of the 65 sequences had a signal peptide; the remaining 10 proteins showed high expression in the MAG. In addition, 29 proteins that were detected in both mated- and unmated-FRT samples also had a signal peptide. These proteins were predicted to be secreted in the MAG. Eventually, a total of 94 proteins were predicted secreted SFPs (Table 2).

One hundred and twenty-four of the 218 proteins had identical conserved domains or were aligned in blastp results with SFPs detected from other insects; this latter class of proteins contained no signal peptide and did not show male-specific expression (Additional file 2: Table S2). Homologues of these proteins had been detected in the SFPs of D. melanogaster, A. aegypti, A. albopictus, or Apis mellifera previously and were classified as unconfirmed SFPs. Whether these proteins are "true" transmitted seminal proteins in $N$. lugens could not be confirmed due to technical limitations (transmitted SFPs could not be distinguished from FRT proteins).

Through sequence annotation, the 218 proteins were classified into different functional groups. By combining protein annotation and the expression patterns of these proteins in the MAG and MRT, we found that predicted secreted SFPs and unconfirmed SFPs exhibited large differences in expression patterns and functional group classifications. MAGs are the main production center for SFPs. The proteins predicted to be secreted showed high expression in the MAG; predicted secreted proteins accounted for $91.10 \%$ of the accumulated RPKM value of the 218 genes. Some proteases, two apolipoprotein D proteins, two cysteine-rich secretory proteins, two peritrophin A-type chitin-binding proteins, three dumpy proteins, a nucleoside diphosphate kinase, a chemosensory protein, and four proteins with unknown function showed extremely high expression in the MAG (RPKM value $>1000$ ). When analyzing the numbers of genes in each functional group, we found that proteolysis regulators represented the largest percentage of secreted proteins (37, $16.97 \%$ ); this was followed by protein modification machinery $(12,5.50 \%)$, proteins with other functions (10, $4.59 \%)$, and lipid metabolism proteins (10, 4.59 \%) (Additional file 3: Table S3 and Fig. 2).

Most of the unconfirmed SFPs showed very low expression levels in the MAG; these genes accounted for only $9.01 \%$ of the accumulated RPKM value of 
Table 2 Identified secreted SFPs of N. lugens

\begin{tabular}{|c|c|c|c|c|c|}
\hline Genes & Function category & D. melanogaster & A. aegypti & A. albopictus & A. mellifera \\
\hline \multicolumn{6}{|l|}{ Cell structure } \\
\hline Annexin & Cell structure & & $\mathrm{T}$ & $\mathrm{T}$ & \\
\hline \multicolumn{6}{|l|}{ Lipid related } \\
\hline Prosaposin & Metabolism: lipid & & & $\mathrm{T}$ & \\
\hline Carboxylesterase (7) & Metabolism: lipid & $\mathrm{T}$ & & & \\
\hline Lysosomal acid lipase & Metabolism: lipid & $\mathrm{T}$ & & & \\
\hline Pancreatic lipase-related protein 2-like & Metabolism: lipid & $\mathrm{T}$ & T & $\mathrm{T}$ & \\
\hline Apolipoprotein D (2) & Transporters and protein export machinery & $\mathrm{T}$ & & & \\
\hline Apolipophorin-III & $\begin{array}{l}\text { Transporters and protein export machinery } \\
\text { and antibacterial }\end{array}$ & & & & \\
\hline \multicolumn{6}{|l|}{ Metabolism } \\
\hline beta-hexosaminidase & & $\mathrm{T}$ & & & \\
\hline Chitinase (2) & Metabolism: carbohydrate & & & & $\mathrm{T}$ \\
\hline Alpha mannosidase & Metabolism: carbohydrate & & T & $\mathrm{T}$ & $\mathrm{T}$ \\
\hline Soluble trehalase & Metabolism: carbohydrate & & & & \\
\hline Nucleoside diphosphate kinase & Metabolism: nucleotide & & T & $\mathrm{T}$ & \\
\hline Carbonic anhydrase & Metabolism: others & & & & \\
\hline \multicolumn{6}{|l|}{ Protein modification machinery } \\
\hline Heat shock 70 kDa protein (2) & Protein modification machinery & & T & $\mathrm{T}$ & $\mathrm{T}$ \\
\hline FKBP-type peptidyl-prolyl cis-trans isomerase (2) & Protein modification machinery & $\mathrm{T}$ & T & & \\
\hline $\begin{array}{l}\text { Gamma-interferon-inducible lysosomal thiol } \\
\text { reductase ( } 2 \text { ) }\end{array}$ & Protein modification machinery & & & $\mathrm{T}$ & $\mathrm{T}$ \\
\hline Protein disulfide isomerase (3) & Protein modification machinery & $\mathrm{T}$ & & $\mathrm{T}$ & \\
\hline Calreticulin & Protein modification machinery & & & $\mathrm{T}$ & \\
\hline $\begin{array}{l}\text { Mesencephalic astrocyte-derived neurotrophic } \\
\text { factor }\end{array}$ & Protein modification machinery & & & & \\
\hline Endoplasmin & Protein modification machinery & & & $\mathrm{T}$ & $\mathrm{T}$ \\
\hline \multicolumn{6}{|l|}{ Proteolysis regulators } \\
\hline Furin-like protease & Proteolysis regulators: protease & & T & $\mathrm{T}$ & \\
\hline Aminopeptidase & Proteolysis regulators: protease & $\mathrm{T}$ & T & $\mathrm{T}$ & $\mathrm{T}$ \\
\hline Zinc carboxypeptidase & Proteolysis regulators: protease & & & & \\
\hline glutamate carboxypeptidase & Proteolysis regulators: protease & & & & \\
\hline angiotensin-converting enzyme & Proteolysis regulators: protease & & T & $\mathrm{T}$ & $\mathrm{T}$ \\
\hline Lysosomal Pro-X carboxypeptidase & Proteolysis regulators: protease & & & & \\
\hline Glutaminyl-peptide cyclotransferase-like & Proteolysis regulators: protease & & & $\mathrm{T}$ & \\
\hline Aspartyl protease (2) & Proteolysis regulators: protease & & & $\mathrm{T}$ & \\
\hline Cathepsin B & Proteolysis regulators: protease & $\mathrm{T}$ & & $\mathrm{T}$ & \\
\hline Serine protease & Proteolysis regulators: protease & & & & \\
\hline Trypsin (20) & Proteolysis regulators: protease & $\mathrm{T}$ & $\mathrm{T}$ & $\mathrm{T}$ & $\mathrm{T}$ \\
\hline SERPIN (2) & Proteolysis regulators: protease inhibitor & $\mathrm{T}$ & T & $\mathrm{T}$ & \\
\hline Kazal type serine protease inhibitors(smart) & Proteolysis regulators: protease inhibitor & & & & $\mathrm{T}$ \\
\hline Cathepsin propeptide inhibitor(smart) & Proteolysis regulators: protease inhibitor & $\mathrm{T}$ & & $\mathrm{T}$ & \\
\hline Pacifastin inhibitor(smart) & Proteolysis regulators: protease inhibitor & & & & \\
\hline Carboxypeptidase inhibitor precursor(blast) & Proteolysis regulators: protease inhibitor & $\mathrm{T}$ & & & \\
\hline
\end{tabular}


Table 2 Identified secreted SFPs of $N$. lugens (Continued)

\begin{tabular}{|c|c|c|c|c|c|}
\hline \multicolumn{6}{|l|}{ Signal transduction } \\
\hline Chemosensory protein (2) & Signal transduction & $\mathrm{T}$ & & & $\mathrm{T}$ \\
\hline Phosphatidylethanolamine binding protein & Signal transduction & $\mathrm{T}$ & $\mathrm{T}$ & & $\mathrm{T}$ \\
\hline Renin receptor & Signal transduction & & & & $\mathrm{T}$ \\
\hline Ion transport peptide & Signal transduction & & & & \\
\hline \multicolumn{6}{|l|}{ Other } \\
\hline Cysteine-rich secretory protein (2) & Other: salivary & $\mathrm{T}$ & $\mathrm{T}$ & $\mathrm{T}$ & $\mathrm{T}$ \\
\hline Unknown & other: oxidoreductase & & & & \\
\hline $\begin{array}{l}\text { Protein with chitin binding Peritrophin-A } \\
\text { domain (4) }\end{array}$ & Other: chitin binding & $\mathrm{T}$ & & & \\
\hline Dumpy(blast) (3) & Other: binding & & & & \\
\hline Selenoprotein & Other: sperm quality & & & & \\
\hline \multicolumn{6}{|l|}{ Unknown } \\
\hline Hypothetical protein(blast) (3) & Unknown & & $\mathrm{T}$ & $\mathrm{T}$ & \\
\hline Unknown (5) & Unknown & & & & \\
\hline
\end{tabular}

Numbers after the gene names stand for the number of proteins detected. Conserved domains of insect SFPs were identifed using the Batch Web CD-Search Tool (http://www.ncbi.nlm.nih.gov/Structure/bwrpsb/bwrpsb.cgi). A local blastp analyse (evalue = 1e-5) were performed between BPH SFPs with other insect SFPs. "T" stands for the sequences possess the same conserved domain or show blastp (1e-5) results with SFPs with other insect SFPs. D. melanogaster, Drosophila melanogaster; A. aegypti, Aedes aegypti; A. albopictus, Aedes. albopictus; A. mellifera, Apis mellifera

the 218 genes. Only several genes encoding cell structure proteins (such as actin, tubulin, myosin, profilin, and tropomyosin), proteins involved in RNA and protein synthesis (such as ribosomal proteins and translation initiation factors), energy metabolism proteins (such as cytochrome c, ATP synthase, and malate dehydrogenase), and other proteins (two thioredoxin genes in particular) (Fig. 2 and Additional file 3: Table S3) had relatively higher expression levels in the MAG. Most of these proteins are ubiquitous

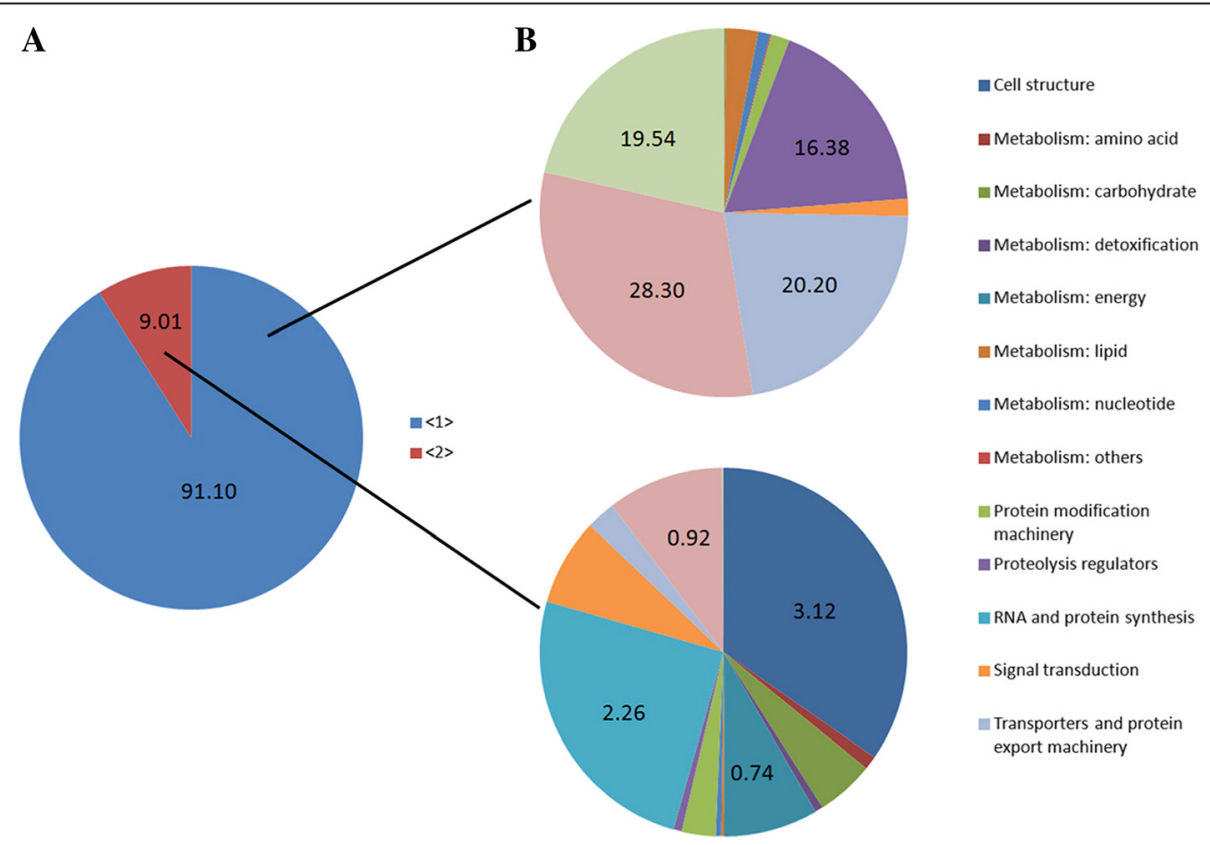

Fig. 2 Functional categories of detected proteins from the MRT and FRT of $N$. lugens. a The percentage of protein expression level in the MAG associated with each group. (The accumulated RPKM value of unigenes in each group/the accumulated RPKM value of the whole unigene). $<1>$ Blue columns, proteins predicted to be secreted SFPs. $<2>$ Red columns, proteins not predicted to be secreted SFPs but that have the same conserved domain or show blastp (1e-5) results with SFPs of D. melanogaster, A. aegypti, A. albopictus, and Apis mellifera seminal fluid. b The percentage of protein expression level in the MAG associated with each functional category. (The accumulated RPKM value of unigenes in a functional group/the accumulated RPKM value of the whole unigene) 
within tissues and throughout the developmental stages. We could not distinguish these proteins from FRT proteins.

One hundred and ninety-six of the 218 proteins had homologues in the SFPs in four other insect species; 73 , 91, 114, and 77 homologous proteins were identified in D. melanogaster, A. aegypti, A. albopictus, and Apis mellifera, respectively. Among the $196 N$. lugens proteins, only trypsins, cysteine-rich secretory proteins, a low-density lipoprotein receptor, and an aminopeptidase were found in all insect species. In $N$. lugens, the lowdensity lipoprotein receptor and the aminopeptidase were not predicted to be secreted; these two proteins also showed very low expression levels in the MAG.

A much larger number of trypsins (20) were detected in $N$. lugens seminal fluid than in other insects $(D$. melanogaster, 10; A. aegypti, 4; A. albopictus, 11; Apis mellifera, 1) (see the phylogenetic tree in Fig. 3). As the phylogenetic tree shows, all $N$. lugens seminal fluid trypsins were on the same branch. Although the SFP genes evolve rapidly, one $D$. melanogaster trypsin showed a closer relationship with $N$. lugens seminal fluid trypsins, and two D. melanogaster trypsin showed a closer relationship with mosquito seminal fluid trypsins. The seminal fluid trypsins from the A. aegypti and A. albopictus were all mixed together. The duplication of $N$. lugens seminal fluid trypsins (including other $N$. lugens SFPs) in the same scaffold was observed (Additional file 2: Table S2). Furthermore, the number of carboxylesterases was also expanded in $N$. lugens. Only one carboxylesterase was identified in the D. melanogaster SFPs, and carboxylesterase was not detected in other insects.

Seven proteins detected in $N$. lugens had not been previously detected in other insect SFPs. These included lysosomal Pro-X carboxypeptidase, carboxypeptidase E, carboxypeptidase Q, prolylcarboxypeptidase, mesencephalic astrocyte-derived neurotrophic factor (MANF), selenoprotein, EGF (epidermal growth factor)-domain containing proteins, and an ion transport peptide-like (ITPL) peptide.

Considering SFPs may undergo enzymatic digestion or other alternations in the female reproductive system, thus, they may not be detected from female samples. Accordingly, we analyzed the signal peptide of the proteins that were only detected from MAG samples. We identified 14 proteins with signal peptides, and their information, including their sequences, is provided in Additional file 4: Table S8.

\section{Expression profile analysis of SFP genes by RT-qPCR}

We tested the expression profiles of 34 genes in the MRT, FRT, and dissections of the MRT using RT-qPCR. Thirty-one genes showed MRT-specific or -biased expression (Additional file 5: Figure S1). Of these 31 genes, we have previously reported that 9 trypsins are specifically expressed in the MRT [29] (not shown in figure). When analyzing the expression of these sequences within the MRT, most of the MRT-specific or -biased genes also showed MAG-specific or -biased expression. Two exceptions were apolipophorin III, which showed the greatest expression in the VD, and a carboxylesterase, which showed high expression in both the VD and the MAG (Additional file 5: Figure S1). The qPCR results were consistent with the gene expression level results of the transcriptome.

\section{Discussion}

The presence of a signal peptide is the classic method of predicting whether a protein is secreted. In D. melanogaster, 142 SFPs were detected; of these, 112 had a signal peptide. Seminal fluid proteome studies in other insects have reported a much lower proportion of proteins with signal peptides (29 of 93 detected proteins in A. aegypti, 57 of 198 detected proteins in A. albopictus, 20 of 53 detected proteins in Apis mellifera). These intracellular or membrane-bound proteins are predicted to be secreted via "apocrine secretion"; cells in the posterior portion of the glands are thought to secrete proteins through granules and/or via the rupture of the cell membrane [18], though some unsolved problems exist concerning "apocrine secretion": 1. The biological importance of these proteins in the female reproductive tract remains to be demonstrated; 2. Proteins may be randomly included in seminal fluid during "apocrine secretion"; 3 . The inclusion in the database of peptide sequences lacking an $\mathrm{N}$-terminus may have complicated the prediction of signal peptides. The high proportion of proteins with a signal peptide in the D. melanogaster SFP proteome may have been due to the high quality of the $D$. melanogaster protein database. In this study, we combined the proteome and expression levels of detected proteins in the MAG and found that proteins with more reliable evidence were secreted (84 proteins with a signal peptide and 10 proteins not detected in unmated female MRT) and showed much higher expression in the MAG than other proteins did. The high expression of these proteins in the MAG is in accordance with the theoretical SFP expression profile.

In D. melanogaster, four trypsins are required in the sex peptide pathway, and C-type lectins are also needed in this pathway. In $N$. lugens, although a large number of trypsins was detected, we did not find C-type lectin in $N$. lugens seminal fluid. Otherwise, astacin family zinc metalloprotease [30], a protein required in fly ovulin (a prohormone-like SFP stimulating ovulation) [31], was not detected in $N$. lugens seminal fluid. In addition, we performed a tblastn using $D$. melanogaster ovulin and sex peptide against $N$. lugens MRT transcriptome 


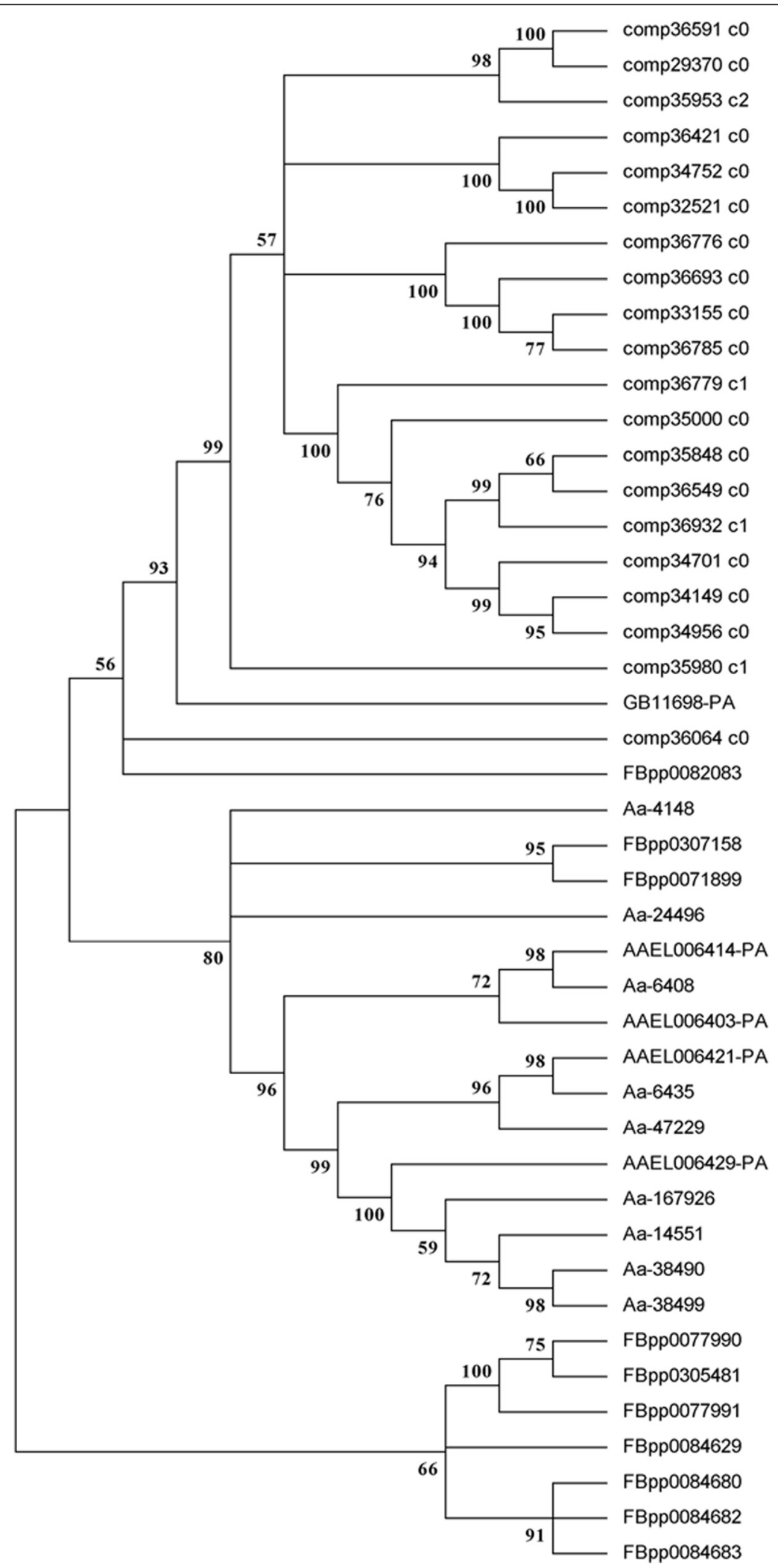

Fig. 3 (See legend on next page.) 
(See figure on previous page.)

Fig. 3 Phylogenetic analysis of insect seminal fluid trypsins. The phylogenetic tree was constructed based on the deduced amino acid sequences of the conserved domains of seminal fluid trypsin genes by maximum likelihood using Mega 5.05 (http://www.megasoftware.net/). The JonesTaylor-Thornton (JTT) model for amino acid substitution was used, while a test of phylogeny was carried out using bootstrap analysis with 1000 replications. Sequences starting with "comp" stand for transcriptome unigene IDs of seminal fluid trypsins in N. lugens. Sequences starting with "FBpp" stand for D. melanogaster genome peptide IDs of seminal fluid trypsins (http://flybase.org/). Sequences starting with "AAEL" stand for A. aegypti genome peptide IDs of seminal fluid trypsins (http://www.vectorbase.org/). Sequences starting with "GB" stand for Apis mellifera genome peptide IDs of seminal fluid trypsins (http://www.ncbi.nIm.nih.gov/). Sequences starting with "Aa" stand for A. albopictus seminal fluid trypsins IDs

unigene sequences, genome coding sequences, and genome DNA sequences, but no homologous sequences were identified. This indicates that the typical sex peptide and ovulin pathway are may not be present in $N$. lugens. The molecular mechanisms behind the postmating phenomena of $D$. melanogaster and $N$. lugens may differ.

An angiotensin-converting enzyme was detected in $N$. lugens: this SFP is critical for increasing female egg laying in T. castaneum [17] and is related to female fecundity in Anopheles stephensi [32]. Two additional proteins associated with angiotensin were also detected in $N$. lugens seminal fluid. One is a renin receptor (detected in $N$. lugens and Apis mellifera) that induces the conversion of angiotensinogen to angiotensin I [33, 34]. The second protein is a lysosomal Pro-X carboxypeptidase (only detected in $N$. lugens) that can cleave $\mathrm{C}$-terminal amino acids linked to proline in peptides such as angiotensin II in response to inactivation $[35,36]$.

Some new insect SFPs were identified in this proteome research, including new seminal fluid proteases, selenoprotein, secreted proteins containing EGF domains, a secreted neurotrophic factor MANF, and a neuropeptide ITPL protein. Selenoproteins were only recently detected in human seminal fluid; they are likely important for protecting sperm during storage [37]. In blastp analysis, secreted proteins containing EGF domains detected in $N$. lugens seminal fluid aligned with dumpy from Drosophila. Dumpy is a huge protein with an EGF domain repeat predicted to be a membrane-anchored fiber almost a micrometer in length; the EGF domain is involved in cell interactions [38]. A secreted protein with an EGF domain was found to bind to the surface of sperm and was important in sperm-egg binding in mice [39]. MANF protects and repairs the dopaminergic neurons. It is up-regulated in response to misfolded proteins, and it protects against various forms of endoplasmic reticulum stress in non-neuronal cells [40].

Most interesting is the discovery of the new seminal fluid neuropeptide, ITPL. IPTs were originally identified in Schistocerca gregaria and are regulators of ion and fluid transport across the ileum. However, ITPL lacks this activity due to C-terminal disparity (Fig. 4). ITP/ITPLs are highly conserved neuropeptides in insects and crustaceans and are grouped into the crustacean hyperglycemic hormones ( $\mathrm{CHH}$ ) [41, 42]. Several insect studies have suggested that ITP functions in ecdysis in Manduca sexta [43] and in D. melanogaster clock neurons [44]. RNAi of ITPL in $T$. castaneum led to significant reduction in egg numbers due to failure in ovarian maturation and reduced survival of offspring after dsRNA injections at the pupal stage [45]. In $N$. lugens, ITPL was identified as a SFP transferred to females after mating. The function of ITPL as an SFP is a topic worth studying.

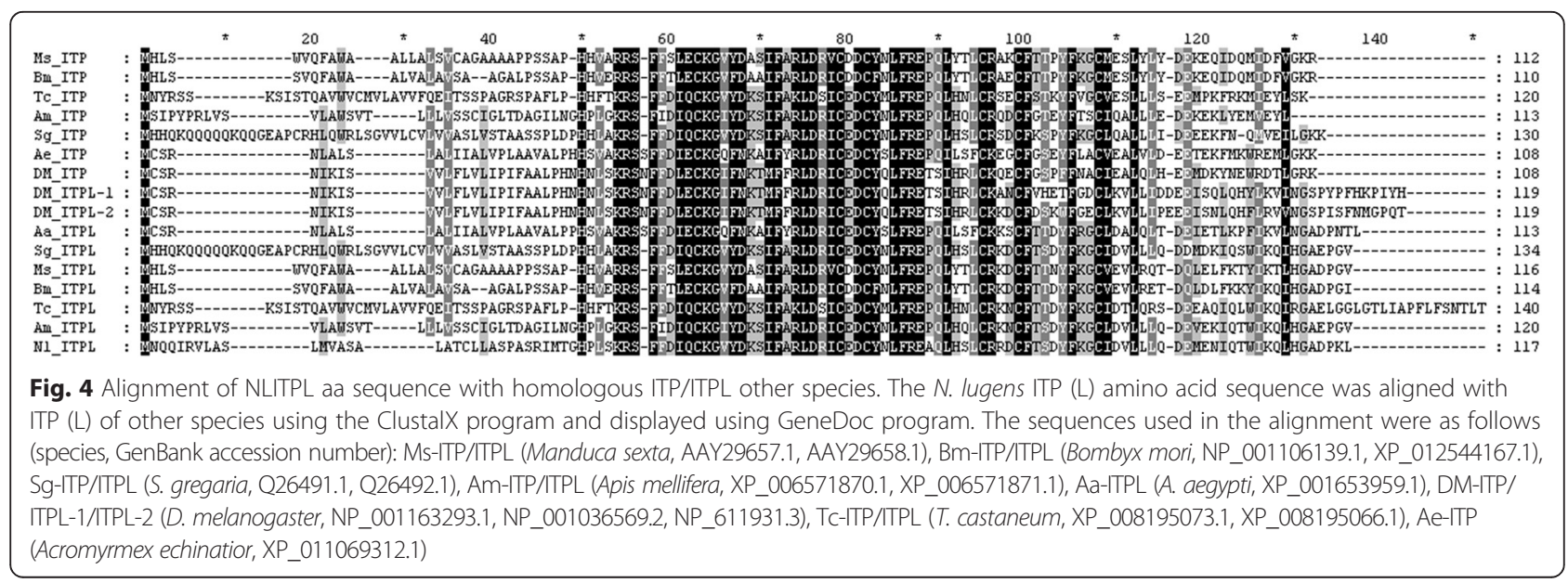




\section{Conclusion}

From the proteomic analysis, we identified 94 putative secreted SFPs of $N$. lugens and the expression level of these proteins in the MAG was yielded by the DGE database. We found that proteins with more reliable evidence predicted to be secreted showed much higher expression in the MAG than other proteins, lending credibility to the detected SFPs. Comparative analyses revealed duplication and expansion of SFPs in $N$. lugens and the identification of novel SFPs in this species. Our results provide a foundation for future studies to investigate the functions of SFPs in N. lugens and are an important addition to the available data for comparative studies of SFPs in insects.

\section{Additional files}

Additional file 1: Table S1. Primers used in this artical. This file lists the sequences of the primers used in the RT-qPCR analysis, as described in Methods. (XLSX $11 \mathrm{~kb}$ )

Additional file 2: Table S2. Detailed annotation of detected proteins. This file gives the detailed annotation of detected proteins including the high confident SFPs and unconfirmed SFPs. The annotation process was described in Methods. (XLSX $134 \mathrm{~kb}$ )

Additional file 3: Table S3. Statistics of function groups. This file gives a statistics analysis of the detected proteins of each function groups, as described in Methods. (XLSX $12 \mathrm{~kb}$ )

Additional file 4: Table S8. Proteins only detected in MAG samples possessing a signal peptide. We analyzed the signal peptide of the proteins that were only detected from MAG samples. We identified 14 proteins with signal peptides, and their information, including their sequences, is provided in this file. (XLSX $31 \mathrm{~kb}$ )

Additional file 5: Figure S1. Analysis of the expression profiles of seminal fluid protein genes in MRT dissections, MRT, and FRT by GRT-PCR. This file gives the RT-qPCR results of the detected proteins. (PDF $613 \mathrm{~kb}$ )

Additional file 6: Table S4. Sequences of detected proteins. This file gives the nucleotide sequences and amino acid sequences of the detected proteins including the high confident SFPs and unconfirmed SFPs. (XLSX $236 \mathrm{~kb}$ )

Additional file 7: Table S5. Comparison of SFPs between different insect species. This file gives the comparison results of SFPs between different insect species, as described in Methods. (XLSX $39 \mathrm{~kb}$ )

Additional file 8: Table S6. Proteome data of each samples. This file gives the proteome raw data of each proteins samples. (XLS $1675 \mathrm{~kb}$ )

Additional file 9: Table S7. Sequences of insect SFPs. This file gives the SFP sequences of other insect species used in the comparison with N. lugens SFPs. (XLSX $502 \mathrm{~kb})$

\section{Funding}

This work was supported by the National Natural Science Foundation of China (grant no.31471765, 31272374).

\section{Availability of data and materials}

The $N$. lugens transcriptomic dataset and gene expression profile datasets used in the male specific expression analysis of SFPs are available in the Sequence Read Archive (SRA) database (http://www.ncbi.nlm.nih.gov/sra). The accession number of the N. lugens transcriptomic dataset is SRX023419. The accession numbers of the $\mathrm{N}$. lugens gene expression profile datasets are as follows: macropterous female adults (MFA) (SRX023495) and macropterous male adults (MMA) (SRX023496). The nucleotide sequences of detected proteins using UPLC/MS/MS (including the predicted secreted SFPS and unconfirmed SFPs) were submitted to National Center for Biotechnology
Information (http://www.ncbi.nlm.nih.gov/). The accession number of these sequences are as follows: KU932205 - KU932419. We also provide the nucleotide and protein sequences of SFPs as an Additional file 6: Table S4 (3 genes with nucleotide sequences length shorter than $200 \mathrm{bp}$ can be found here, they can't be submitted to GenBank due to short length). The phylogenetic data was uploaded to Dryad (http://purl.org/phylo/treebase/ phylows/study/TB2:S19675). The annotation and gene expression profile in MRT and MAG of detected proteins were provided in Additional file 2: Table S2. Comparison of SFPs between different insect species were provided in Additional file 7: Table S5. The proteome raw data of each proteins samples were provided as Additional file 8: Table S6. The SFP sequences of other insect species used in the comparison with $N$. lugens SFPs were provided as Additional file 9: Table S7.

\section{Authors' contribution}

ZCX conceived the experiments. BY designed the experiments and wrote the manuscript. DTL, JBL and ZWX helped to perform the experiments. All authors discussed the results and commented on the manuscript. All authors read and approved the final manuscript.

\section{Competing interests}

The authors declare that they have no competing interests.

\section{Consent for publication}

Not applicable.

Ethics approval and consent to participate

Not applicable.

Received: 22 January 2016 Accepted: 12 August 2016

Published online: 18 August 2016

\section{References}

1. LaFlamme BA, Wolfner MF. Identification and function of proteolysis regulators in seminal fluid. Mol Reprod Dev. 2013;80(2):80-101.

2. Poiani A. Complexity of seminal fluid: a review. Behav Ecol Sociobiol. 2006; 60(3):289-310

3. Andres JA, Maroja LS, Bogdanowicz SM, Swanson WJ, Harrison RG. Molecular evolution of seminal proteins in field crickets. Mol Biol Evol. 2006;23(8):1574-84

4. Gillott C. Male accessory gland secretions: Modulators of female reproductive physiology and behavior. Annu Rev Entomol. 2003;48:163-84

5. Ram KR, Wolfner MF. Seminal influences: Drosophila Acps and the molecular interplay between males and females during reproduction. Integr Comp Biol. 2007:47(3):427-45.

6. Ram KR, Wolfner MF. Sustained post-mating response in Drosophila melanogaster requires multiple seminal fluid proteins. Plos Genet. 2007; 3:2428-38.

7. Thailayil J, Magnusson K, Godfray HCJ, Crisanti A, Catteruccia F. Spermless males elicit large-scale female responses to mating in the malaria mosquito Anopheles gambiae. Proc Natl Acad Sci U S A. 2011; 108(33):13677-81

8. Baer B, Heazlewood JL, Taylor NL, Eubel H, Millar AH. The seminal fluid proteome of the honeybee Apis mellifera. Proteomics. 2009;9(8): 2085-97.

9. den Boer SPA, Baer B, Boomsma JJ. Seminal Fluid Mediates Ejaculate Competition in Social Insects. Science. 2010;327(5972):1506-9.

10. Xue J, Zhou X, Zhang C, Yu L, Fan H, Wang Z, Xu H, Xi Y, Zhu Z, Zhou W, et al. Genomes of the rice pest brown planthopper and its endosymbionts reveal complex complementary contributions for host adaptation. Genome Biol. 2014;15:521.

11. Long $Y$, Hou ML, Yang $X$, Shi BK. Effects of delayed mating on reproduction in brachypterous females of the brown planthopper, Nilaparvata lugens. Plant Prot. 2010;6:36-9.

12. Ichikawa T. Studies on the mating behavior of the four species of auchenorrhynchous Homoptera which attack the rice plant. Memoirs of Faculty of Agriculture Kagawa University. 1979.

13. Xue J, Bao Y, Li B, Cheng Y, Peng Z, Liu H, Xu H, Zhu Z, Lou Y, Cheng J, et al. Transcriptome Analysis of the Brown Planthopper Nilaparvata lugens. Plos One. 2010;5(12):e14233. doi:10.1371/journal.pone.0014233. 
14. Ge L, Wu J, Zhao K, Chen Y, Yang G. Induction of Nlvg and suppression of Nljhe gene expression in Nilaparvata lugens (Stal) (Hemiptera: Delphacidae) adult females and males exposed to two insecticides. Pestic Biochem Phys. 2010;98(2):269-78.

15. Wang L, Shen J, Ge L, Wu J, Yang G, Jahn GC. Insecticide-induced increase in the protein content of male accessory glands and its effect on the fecundity of females in the brown planthopper Nilaparvata lugens Sta (Hemiptera: Delphacidae). Crop Prot. 2010;29(11):1280-5.

16. Findlay GD, Yi X, MacCoss MJ, Swanson WJ. Proteomics reveals novel Drosophila seminal fluid proteins transferred at mating. Plos Biol. 2008; 6(e1787):1417-26.

17. Xu J, Baulding J, Palli SR. Proteomics of Tribolium castaneum seminal fluid proteins: Identification of an angiotensin-converting enzyme as a key player in regulation of reproduction. J Proteomics. 2013;78:83-93.

18. Sirot LK, Hardstone MC, Helinski MEH, Ribeiro JMC, Kimura M, Deewatthanawong P, Wolfner MF, Harrington LC. Towards a Semen Proteome of the Dengue Vector Mosquito: Protein Identification and Potential Functions. Plos Neglect Trop D. 2011;5(3):e989.

19. Boes KE, Ribeiro JMC, Wong A, Harrington LC, Wolfner MF, Sirot LK. Identification and Characterization of Seminal Fluid Proteins in the Asian Tiger Mosquito, Aedes albopictus. Plos Neglect Trop D. 2014;8(6):e2946.

20. Simmons LW, Tan YF, Millar AH. Sperm and seminal fluid proteomes of the field cricketTeleogryllus oceanicus: identification of novel proteins transferred to females at mating. Insect Mol Biol. 2013;22(1):115-30.

21. Walters JR, Harrison RG. Combined EST and Proteomic Analysis Identifies Rapidly Evolving Seminal Fluid Proteins in Heliconius Butterflies. Mol Biol Evol. 2010;27(9):2000-13.

22. Avila FW, Sirot LK, LaFlamme BA, Rubinstein CD, Wolfner MF. Insect Seminal Fluid Proteins: Identification and Function. Annu Rev Entomol. 2011;56:21-40.

23. Bao Y, Wang Y, Wu W, Zhao D, Xue J, Zhang B, Shen Z, Zhang C. De novo intestine-specific transcriptome of the brown planthopper Nilaparvata lugens revealed potential functions in digestion, detoxification and immune response. Genomics. 2012;99(4):256-64.

24. Ji R, Yu H, Fu Q, Chen H, Ye W, Li S, Lou Y. Comparative Transcriptome Analysis of Salivary Glands of Two Populations of Rice Brown Planthopper, Nilaparvata lugens That Differ in Virulence. Plos One. 2013;8:e7961211.

25. Tanaka Y, Suetsugu Y, Yamamoto K, Noda H, Shinoda T. Transcriptome analysis of neuropeptides and G-protein coupled receptors (GPCRs) for neuropeptides in the brown planthopper Nilaparvata lugens. Peptides. 2014;53:125-33.

26. Yu H, Ji R, Ye W, Chen H, Lai W, Fu Q, Lou Y. Transcriptome Analysis of Fat Bodies from Two Brown Planthopper (Nilaparvata lugens) Populations with Different Virulence Levels in Rice. Plos One. 2014;9:e885282.

27. Wisniewski JR. Quantitative Evaluation of Filter Aided Sample Preparation (FASP) and Multienzyme Digestion FASP Protocols. Anal Chem. 2016;88(10): 5438-43.

28. Pilch B, Mann M. Large-scale and high-confidence proteomic analysis of human seminal plasma. Genome Biol. 2006;7:R405.

29. Bao Y, Qin X, Yu B, Chen L, Wang Z, Zhang C. Genomic insights into the serine protease gene family and expression profile analysis in the planthopper Nilaparvata lugens. BMC GENOMICS. 2014;15:507.

30. Ravi Ram K, Sirot LK, Wolfner MF. Predicted seminal astacin-like protease is required for processing of reproductive proteins in Drosophila melanogaster. Proc Natl Acad Sci U S A. 2006;103(49):18674-9.

31. Heifetz Y, Lung O, Frongillo EA, Wolfner MF. The Drosophila seminal fluid protein Acp26Aa stimulates release of oocytes by the ovary. Curr Biol. 2000; 10(2):99-102.

32. Ekbote U, Looker M, Isaac RE. ACE inhibitors reduce fecundity in the mosquito Anopheles stephensi. Comp Biochem Phys B. 2003;134(4):593-8.

33. Oshima $Y$, Morimoto $S$, Ichihara A. Roles of the (pro)renin receptor in the kidney. World J Urol. 2014;3:302-7.

34. Cousin C, Bracquart D, Contrepas A, Corvol P, Muller L, Nguyen G. Soluble Form of the (Pro) Renin Receptor Generated by Intracellular Cleavage by Furin Is Secreted in Plasma. Hypertension. 2009;53(6):1077-82.

35. Xu S, Lind L, Zhao L, Lindahl B, Venge P. Plasma Prolylcarboxypeptidase (Angiotensinase C) Is Increased in Obesity and Diabetes Mellitus and Related to Cardiovascular Dysfunction. Clin Chem. 2012;58(7):1110-5.

36. Tan F, Morris PW, Skidgel RA, Erdös EG. Sequencing and cloning of human prolylcarboxypeptidase (angiotensinase C). Similarity to both serine carboxypeptidase and prolylendopeptidase families. J Biol Chem. 1993;268: 26032-8.
37. Michaelis M, Gralla O, Behrends T, Scharpf M, Endermann T, Rijntjes E, Pietschmann N, Hollenbach B, Schomburg L. Selenoprotein P in seminal fluid is a novel biomarker of sperm quality. Biochem Bioph Res Co. 2014; 443(3):905-10.

38. Wilkin MB, Becker MN, Mulvey D, Phan I, Chao A, Cooper K, Chung HJ, Campbell ID, Baron M, Maclntyre R. Drosophila Dumpy is a gigantic extracellular protein required to maintain tension at epidermal-cuticle attachment sites. Curr Biol. 2000;10(10):559-67.

39. Shur BD, Ensslin MA, Rodeheffer C. SED1 function during mammalian sperm-egg adhesion. Curr Opin Cell Biol. 2004;16(5):477-85.

40. Yang W, Shen Y, Chen Y, Chen L, Wang L, Wang H, Xu S, Fang S, Fu Y, Yu Y, et al. Mesencephalic astrocyte-derived neurotrophic factor prevents neuron loss via inhibiting ischemia-induced apoptosis. J Neurol Sci. 2014;344(1-2): 129-38.

41. Audsley N, McIntosh C, Phillips JE. Isolation of a neuropeptide from locust corpus cardiacum which influences ileal transport. J Exp Biol. 1992;173:261-74

42. Meredith J, Ring M, Macins A, Marschall J, Cheng NN, Theilmann D, Brock HW, Phillips JE. Locust ion transport peptide (ITP): Primary structure, CDNA and expression in a baculovirus system. J Exp Biol. 1996;199:1053-61.

43. Drexler AL, Harris CC, Dela Pena MG, Asuncion-Uchi M, Chung S, Webster S, Fuse M. Molecular characterization and cell-specific expression of an ion transport peptide in the tobacco hornworm Manduca sexta. Cell Tissue Res. 2007:329(2):391-408.

44. Hermann-Luibl C, Yoshii T, Senthilan PR, Dircksen H, Helfrich-Foerster C. The Ion Transport Peptide Is a New Functional Clock Neuropeptide in the Fruit Fly Drosophila melanogaster. J Neurosci. 2014;34(29):9522-36.

45. Begum K, Li B, Beeman RW, Park Y. Functions of ion transport peptide and ion transport peptide-like in the red flour beetle Tribolium castaneum. Insect Biochem Molec. 2009;39(10):717-25.

\section{Submit your next manuscript to BioMed Central and we will help you at every step:}

- We accept pre-submission inquiries

- Our selector tool helps you to find the most relevant journal

- We provide round the clock customer support

- Convenient online submission

- Thorough peer review

- Inclusion in PubMed and all major indexing services

- Maximum visibility for your research

Submit your manuscript at www.biomedcentral.com/submit 